\title{
Una lesión en el cintigrama renal DMSA 6 meses post fase aguda de una pielonefritis representa siempre una cicatriz: un debate abierto
}

\author{
GILDA DONOSO $^{1}$, ELIZABETH LAGOS ${ }^{2}$, PÍA ROSATI ${ }^{2}$, \\ PILAR HEVIA ${ }^{2}$, KAREN CUEVAS ${ }^{2}$, GABRIEL LOBO ${ }^{1}$, ANDRÉS PÉREZ ${ }^{1, a}$, \\ CÉSAR JIMÉNEZ ${ }^{1, a}$, DANIELA GUTIÉRREZ ${ }^{1}$
}

'Servicio de Medicina

Nuclear, Hospital San Juan de Dios-Universidad de

Chile.

${ }^{2}$ Servicio de Nefrología Infantil, Hospital San Juan de Dios.

Tecnólogo Médico.

Recibido el 14 de julio de 2011, aceptado el 17 de enero de 2012.

Correspondencia a: Dra. Gilda Donoso R. Servicio Medicina Nuclear CDT-Hosp. San Juan de Dios.

Portales 3239, Santiago. Fono-Fax: 5742015

E-mail: gdonosor@yahoo.

\section{Evolution of scintigraphic renal lesions in children after an episode of acute pyelonephritis}

Background: Abnormal Dimercaptosuccinic acid (DMSA) renal scintigraphy performed six months after an acute pyelonephritis $(A P)$ is generally interpreted as scarring. Aim: To perform a follow up of childhood patients showing scintigraphic renal lesions during the acute phase of pyelonephritis (within 7 days from the beginning of fever). Material and Methods: A scintigraphic control was carried out at 5-7 months and, in case of persistent lesions, an additional late scintigraphy at 10-13 months. All patients were followed clinically for one year and those with a relapse of urinary tract infection were excluded from the study. Results: Eighty five patients with a median age of 8 months were included. Among these, the first scintigraphic control was normal in 59 (69\%) and abnormal in 26 patients (31\%). In five of these 26 patients (5/26: 19\%-5/85: 6\%), a considerable regression of the lesions was obvious on the early control, and normalized completely on the late control. When expressing the results in kidney units, 107 showed lesions during the acute phase of infection; 69\% was normal at the early control. Thirty three showed lesions persisting at the early control (31\%) and 7 out of these $33(21 \%)$ became normal on the late control (7/107: 7\%). In total, $25 \%$ of the children included in the study (24\% of the kidney units) remained with renal sequelae one year after the initial episode of AP. Conclusions: The persistence of scintigraphic lesions six months after an episode of AP, does not necessarily correspond to permanent scars, since normalization can sometimes be observed on late controls.

(Rev Med Chile 2012; 140: 746-750).

Key words: Child; Pyelonephritis; Radionuclear imaging.
$\mathrm{E}$ 1 cintigrama renal con ácido dimercapto succínico (CR DMSA) es un estudio de imágenes de Medicina Nuclear con baja irradiación para el paciente, ampliamente conocida por su sensibilidad en la detección de compromiso de parénquima renal en niños con pielonefritis, tanto en la fase aguda de la enfermedad como en el seguimiento del compromiso renal ${ }^{1,2}$.

Se ha asociado la existencia de estas cicatrices renales con el desarrollo posterior de hipertensión arterial, insuficiencia renal o ambos ${ }^{3}$, por lo que este grupo de pacientes debe ser especialmente controlado para detectar precozmente una complicación.

A pesar de que existe controversia en la utilización del CR DMSA en el diagnóstico de una pielonefritis aguda (PA), sí existe consenso en su utilidad para el seguimiento de estos niños y la 
evaluación de la existencia de secuelas renales ${ }^{4,5}$. La discusión existe aún sobre el momento apropiado para realizar el examen y determinar la presencia de esta cicatriz.

En el estudio rutinario de nuestros pacientes observamos que en algunos de ellos se veía una normalización más tardía de la(s) lesión(es) renales, lo que motivó este trabajo prospectivo. Mantener un niño asintomático en control médico periódico y a sus padres tranquilos puede ser una tarea difícil. Por esto importa conocer el tiempo necesario que debe transcurrir luego del cuadro infeccioso agudo para estar seguros de la existencia de secuelas renales permanentes.

\section{Material y Método}

Los pacientes fueron estudiados según protocolo de nuestro Servicio para niños admitidos con diagnóstico clínico de primera pielonefritis aguda, con ecotomografía renal y vesical y uretrocistografía radiológica. Si no se disponía de esta última técnica, se realizó una cistografía isotópica directa.

Todos ellos recibieron tratamiento inicial con cefradina i.v., y completaron tratamiento con cefadroxilo oral por un total de 10 días. Posteriormente, se indicó profilaxis antibiótica por 6 meses.

Se excluyeron del estudio a aquellos pacientes en los que durante su estudio se les diagnosticaron malformaciones renales como ectopías, riñones en herradura, hidronefrosis, doble sistema excretor con o sin ureterocele, manteniendo en el estudio aquellos pacientes con reflujo vesicoureteral (RVU). También fueron excluidos todos aquellos que presentaron un nuevo episodio de infección urinaria (alta o baja) durante el seguimiento.

El CR DMSA se realizó dentro de los 7 días de iniciada la fiebre con técnica habitual ${ }^{8}$ en Gammacámara Picker Dyna, asociada a computador Alfa Nuclear. La dosis de Tc99m DMSA se administró según tabla sugerida por la European Association of Nuclear Medicine 9 . Se obtuvieron imágenes planares de 300.000 a 500.000 cuentas, realizadas $2-4 \mathrm{~h}$ post administración del radiotrazador (punción i.v.) en proyecciones póstero-anterior, oblicua posterior derecha e izquierda y antero-posterior; usando un colimador multipropósito. Se realizó el cálculo de función relativa de cada riñón ${ }^{2}$.

Cada CR DMSA fue evaluado por 2 especialistas en Medicina Nuclear con experiencia en el área nefrourológica y fue catalogado como anormal cuando existían 1 o más defectos de contraste corticales y/o disminución de contraste global y/o alteración en el tamaño renal y/o disminución de la función renal relativa ( Valor normal de función relativa: $50 \pm 5 \%$ ).

El CR DMSA fue realizado en la fase aguda de la pielonefritis, dentro de los 7 días de inicio de la fiebre y luego se obtuvo un control precoz 5-7 meses post cuadro clínico. En aquellos en que persistían lesiones cintigráficas se solicitó control tardío (10-13 meses post PA). El tiempo entre el control precoz y el tardío fue al menos de 5 meses.

\section{Resultados}

Completaron el seguimiento 85 pacientes con un total de 107 (UR) alteradas (22 pacientes con compromiso bilateral).

De ellos, 55 fueron mujeres (65\%); mediana edad: 8 meses (rango: 11 días-10 años).

Se observó RVU en 9 pacientes de los 68 que se habían realizado el estudio (13\%): 8 con RVU grados I-II ( 2 bilaterales) y 1 con RVU grado V bilateral.

En el CR a los 6 meses, 26 pacientes (31\%) persistían con anormalidades (33/107 UR : 31\%) y en 59 pacientes $(69 \%)$ se observó normalización (74/107 UR: 69\%) (Figura 1 y Tabla 1).

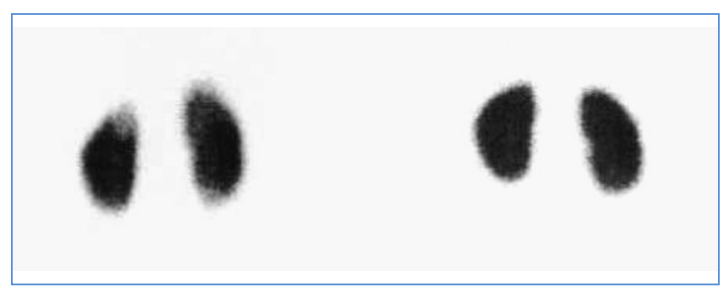

Figura 1. DMSA agudo y control a los 6 meses.

Tabla 1.

\begin{tabular}{|lccc|}
\hline & $\begin{array}{c}\text { DMSA } \\
\text { alterado } \\
\text { Fase aguda }\end{array}$ & $\begin{array}{c}\text { DMSA } \\
\text { alterado } \\
\mathbf{6} \text { meses }\end{array}$ & $\begin{array}{c}\text { DMSA } \\
\text { alterado } \\
\mathbf{1} \text { año }\end{array}$ \\
Pacientes & 85 & 26 & 21 \\
$\begin{array}{l}\text { Unidades } \\
\text { renales }\end{array}$ & 107 & 33 & 26 \\
\hline
\end{tabular}




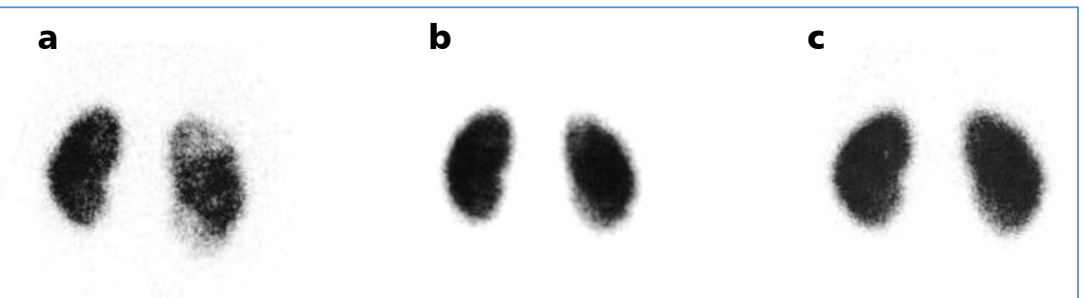

Figura 2. a) DMSA agudo con alteraciones de riñón derecho; b) DMSA precoz con regresión parcial; c) DMSA tardío con normalización de lesiones.
De los 26 pacientes que persistían con alteraciones a los 6 meses, 5 se normalizaron en el control tardío: 5/26: 19\%; 5/85 (total de pacientes): 6\%. En UR: 7 de 33 se normalizaron en el control tardío: 7/33:21\%; 7/107: 7\%. Los mayores cambios se observaron en los primeros 6 meses (Figura 2).

En 21 de los 85 pacientes en seguimiento (25\%) se observó alteración cintigráfica aún en el control tardío (26/107 UR: 24\%).

\section{Discusión}

La PA es una patología de difícil diagnóstico en los niños, especialmente lactantes, que presentan un cuadro clínico inespecífico. La importancia del diagnóstico rápido radica en que algunos grupos han demostrado que el riesgo de desarrollar daño renal permanente se relaciona con la prontitud o tardanza con que se inicia el tratamiento antibiótico $^{5,7,10}$.

El uso del CR DMSA en la fase aguda de la PA es discutido, ya que su resultado no cambiará el manejo terapéutico por parte de la mayoría de los médicos tratantes, pero sirve para identificar la población de riesgo de desarrollar una cicatriz. Es sabido que un paciente con CR DMSA normal realizado en la fase aguda de una PA tiene $0 \%$ de riesgo de desarrollar una cicatriz ${ }^{11}$.

En lo que existe consenso es en su utilidad para estudiar la existencia de lesiones renales permanentes, con una sensibilidad muy superior al ultrasonido con Doppler ${ }^{12,13}$. La pielografía iv y la tomografía computada son técnicas con reconocida resolución anatómica para evidenciar daño renal pero debido a su alto nivel de irradiación no está indicado su uso habitual en la población pediátrica $^{14-17}$.

Mucha de la literatura publicada considera que 6 meses post episodio agudo de la PA es el tiempo adecuado para evaluar la presencia de cicatriz con un CR DMSA, incluso algunos grupos realizan controles a los 3 meses $^{4,18,19}$. Este concepto no es avalado por nuestros hallazgos.

Los resultados generales obtenidos nos muestran, en primer lugar, que nuestra población con PA no difiere en forma significativa de los pacientes incluidos en otros estudios publicados en cuanto a edades, porcentaje de secuelas y la baja asociación con RVU ${ }^{18-20}$. Actualmente, es muy cuestionada la presencia de RVU como factor de riesgo de cicatriz renal, aunque sí existen evidencias de la relación significativa entre daño renal permanente y RVU de alto grado (III-V $)^{21}$. Sólo uno de nuestros pacientes tenía RVU grado $\mathrm{V}$ y se asoció a cicatriz renal.

En nuestra población la mayoría de las lesiones cintigráficas se normalizan aproximadamente a los 6 meses de seguimiento, existiendo también una importante regresión en la intensidad, tamaño y/o número de lesiones en los demás. Las características de las alteraciones cintigráficas podrían ser revisadas posteriormente para evidenciar su relación con el riesgo de desarrollar cicatriz.

Siete de 33 UR, 5 de 26 pacientes ( $21 \%$ y $19 \%$ respectivamente), se normalizan entre los 6 meses y el año. En todos ellos, ya en el control cintigráfico precoz, se había observado una gran disminución de las alteraciones descritas inicialmente. Nuestro hallazgo difiere de lo descrito en un estudio realizado por Agras y cols en que describen que un alto número de sus pacientes se normalizan entre los 6 meses y el año ${ }^{4}$.

El CR DMSA es, actualmente, el gold standard para el diagnóstico de secuela renal de una PA. Es una técnica con buena reproducibilidad inter e intra observador ${ }^{22}$ siempre que el examen sea realizado en forma apropiada, con imágenes de buena resolución y adecuada técnica, ya que algunas, mal interpretadas como lesiones cintigráficas, pueden corresponder a artefactos técnicos. Es importante también el conocimiento de las variantes normales de los riñones para disminuir los falsos positivos. 
Estos son algunos de los factores que influyen en los diferentes resultados obtenidos en distintas series de pacientes ${ }^{2}$.

En el último tiempo se está discutiendo la posibilidad de no realizar el estudio de RVU ante una primera PA con CR DMSA agudo normal, considerando que numerosas revisiones, incluida la nuestra, demuestran que en un bajo número de pacientes con este cuadro clínico existe RVU asociado $^{20}$. Son necesarias aún más estudios controlados para llegar a un consenso y así evitar la invasividad en el estudio inicial de estos pacientes.

Nuestros hallazgos son concordantes con lo descrito en la literatura, ya que la mayor parte de las lesiones agudas desaparecen en el control precoz (6 meses aproximadamente), por lo que un examen realizado en este tiempo sería útil para evidenciar la normalización renal. Si en este control la o las lesión (es) persiste (n) será necesario un control más tardío ${ }^{4}$.

En términos estrictos, los pacientes con CR DMSA agudo anormal en una PA, si no vuelven a presentar un cuadro infeccioso urinario alto o bajo, deberían ser controlados al año post infección para evaluar la existencia de cicatriz. El problema es que mientras más largo el período de seguimiento más posibilidades de deserción de los pacientes a los controles. Por eso creemos que un examen a los 6 meses es adecuado, ya que en una gran parte de los casos las lesiones habrán desaparecido y los pacientes podrán ser dados de alta de sus controles. Si a los 6 meses se observan aún lesiones, será necesario un control más tardío.

\section{Conclusiones}

Una lesión renal visible en un CR DMSA realizado dentro de los 6 meses de una pielonefritis aguda no corresponde necesariamente a una cicatriz, por lo que es necesario realizar un control tardío (1 año).

\section{Referencias}

1. Piepsz A. Cortical scintigraphy and urinary tract infection in children. Nephrol Dial Transplant 2002; 17: 560-2.

2. Piepsz A, Blaufoux Md, Gordon I, Granerus G, Majd M, O’Really P, et al. Consensus on renal cortical scintigra- phy in children with urinary tract infection. Sem Nucl Med 1999; 2: 160-74.

3. Jacobson SH, Eklof O, Eriksson CG, Lins LE, Tidgren B, Winberg J. Development of hypertension and uraemia after pyelonephritis in childhood: 27 year followup. BMJ 1989; 299: 703-6.

4. Koray Agras R, Ortapamuk H, Naldöken S, Tuncel A, Atan A. Resolution of cortical lesions on serial renal scans in children with acute pyelonephritis. Pediatr Radiol 2007; 37: 153-8.

5. Biassoni L, Chippington S. Imaging in Urinary Tract Infections: Current Strategies and New Trends. Semin Nucl Med 2008; 38: 56-66.

6. Jodal U, Lindberg U, Lincoln $\mathrm{K}$. Level diagnosis of symptomatic urinary tract infections in childhood. Acta Paediatr Scand 1975; 64: 201-8.

7. American Academy of Pediatrics. Committee on Quality Improvement. Subcommittee on urinary tract infections. Practice parameter: the diagnosis, treatment and the evaluation of the initial urinary tract infection in febrile infants and young children. Pediatrics 1999; 103: 843-52.

8. Piepsz A, Colarinha P, Gordon I, Hahn K, Olivier P, Roca I, et al. Guidelines for $99 \mathrm{mTc}$-DMSA scintigraphy in children. Eur J Nucl Med 2001; 28: BP15-BP47.

9. www.eanm.org/committees/dosimetry/dosagecard.pdf

10. Geier P, Strojil J, Kutrová K. Therapeutic Delay in Infant Urinary Tract Infection: Does It Really Have No Impact?: Letter to the Editor. Pediatrics 2008; 122: 215-6.

11. Rushton HG, Majd M. DMSA scintigraphy for the evaluation of pyelonephritis and scarring: a review of experimental and clinical studies. J Urol 1992; 148 (5pt. 2): 1726-32.

12. Je Mo Yoo, Jun Sung Koh, Chang Hee Han, Su Lim Lee, U-Syn Ha, Sung Hak, et al. Diagnosing Acute Pyelonephritis with CT, ${ }^{99 \mathrm{~m} T c-D M S A ~ S P E C T}$, and Doppler Ultrasound: A Comparative Study. Korean J Urol 2010; 51 (4): 260-5.

13. Moorthy I, Wheat D, Gordon I. Ultrasonography in the evaluation of renal scarring using DMSA scan as the gold standard. Pediatr Nephrol 2004; 19: 153-6.

14. Hansen A, Wagner AA, Lavard LD, Nielsen JT. Diagnostic imaging in children with urinary tract infection: the role of intravenous urography. Radiology 2009; 250 (2): 309-23.

15. Padmakumar B, Carty HM, Hughes DA, Judd BA. Role of intravenous urogram in investigation of urinary tract infection: an observational study. Postgrad Med J 2004; 80:424-425. doi: 10.1136/pgmj.2003.011148.

16. Silverman SG, Leyendecker JR, Amis ES JR. What is the current role of CT urography and MR urography in the 
evaluation of the urinary tract? Radiology 2009; 250 (2): 309-23.

17. Mondaca R. Por qué reducir las dosis de radiación en pediatría. Revista Chilena de Radiología 2006; 12: 28-32.

18. Orellana P, Baquedano P, Rangarajan V, Jin Hua Zhao NG, David Chee Eng J, Fettich J, et al. Relationship between acute pyelonephritis, renal scarring, and vesicoureteral reflux. Pediatr Nephrol 2004; 19: 1122-6.

19. Faust W, Díaz M, Pohl H. Incidence of Post-Pyelonephritic Renal Scarring: A Meta-Analysis of the Dimercapto-Succinic Acid Literature. The Journal of Urology 2009; 181: 290-8.

20. Donoso G, Lobo G, Arnello F, Arteaga P, Hevia P, Rosati
P, et al. Cintigrama renal DMSA en niños con primera pielonefritis aguda: correlación con exámenes de laboratorio, ecografía y la presencia de reflujo vésico ureteral. Rev Med Chile 2004; 132: 58-64.

21. Hansson S, Dhamey M, Sigström O, Sixt R, Stokland E, Wennerström $M$, et al. Dimercapto-succinic acid scintigraphy instead of voiding cystourethrography for infants with urinary tract infection. J Urol 2004; 172: 1071-3.

22. Ladrón de Guevara D, Franken PH, De Sadeleer C, Ham $\mathrm{H}$, Piepsz A. Interobserver Reproducibility in Reporting on $99 \mathrm{mTc}$-DMSA Scintigraphy for Detection of Late Renal Sequelae. J Nucl Med 2001; 42: 564-6. 